\title{
Gender disparities and thrombolysis use among patient with first-ever ischemic stroke in Malaysia
}

\begin{abstract}
Objective: Gender as an independent predictor in stroke has been well documented. However, data on gender differences among first-ever ischemic stroke in developing country are limited. We aim to describe gender effects on clinical characteristics, thrombolysis treatment received, and outcomes of patients with first-ever ischemic stroke. Methods: Data were extracted from the prospective multiethnic stroke registry, National Neurology Registry (NNEUR). Descriptive analysis and logistic regression were performed. Results: A total of 4762 first-ever ischemic stroke patients admitted to 13 government hospitals from July 2009 to June 2015 were available for this study. Slightly over half were male (55.1\%), and they were 1.7 years younger than female (mean age, 63.6 versus 61.9 years, $p<0.001$ ). Genderage-adjusted incidence was observed to be higher in females $(66.7$ per 100,000$)$ compared to males (57.4 per 100,000). First-ever ischemic stroke incidence increased by 24.3 and $11.2 \%$ among female and male annually. Female experienced significantly poorer functional outcome and greater 30-day in-hospital mortality compared to male. In subgroup analysis, only $31(0.65 \%)$ patients were treated with thrombolysis. Discussion: First-ever ischemic stroke incidence increased by 24.3 and $11.2 \%$ among female and male annually. There were distinct symptoms at hospital presentation between genders. All our patients discharged home regardless of genders. In summary, Malaysian female first-ever ischemic stroke was older, present with severe stroke, greater number of risk factors and poorer functional outcome and 30-day in-hospital mortality compared to male.
\end{abstract}

Keyword: Gender; Disparities; Predictor; Clinical; Registry; Ischemic 